\title{
Stochastic modulation of non-Abelian gauge potential. Spin-rotational relaxation
}

\author{
Yu.A. Serebrennikov, U.E. Steiner \\ Fakultät für Chemie, Universität Konstanz, D-78434 Konstanz, Germany
}

\begin{abstract}
The process of non-Abelian gauge field modulation in systems undergoing cyclic chaotic evolution in the parameter space is treated in terms of the stochastic Liouville formalism. It is shown that spin-rotational relaxation in systems with an effective electronic spin $S_{\mathrm{ef}}=1 / 2$ arises from the stochastic modulation of a $\mathrm{U}(2)$ non-Abelian gauge potential.
\end{abstract}

\section{Introduction}

It is now well recognized that topology plays a fundamental role in all forces of nature. The intensive 'gcometrization' of some areas of chemical physics was stimulated by Berry's remarkable discovery of a geometric phase in a non-degenerate quantum system coupled to a slowly changing (cycling) environment [1]. Due to the generality of the geometrical approach many old problems appear in a new light [2].

A fundamental study of the geometric phase for systems whose classical dynamics is chaotic was made by Robbins and Berry [3]. Performing an average over the 'fast chaos' [3] of the system for fixed external parameters they obtained the geometric vector potential (U(1) gauge symmetry) in a form applicable to systems whose intrinsic dynamics are chaotic.

Quite a different problem, namely the calculation of Berry's phase in the presence of a chaotic external forcing (stochastically modulated external parameters) has been considered by Gamliel and Freed [4]. Their consideration was based on the stochastic Liouville-type equation constructed by adding to the
Liouville-von Neumann equation of motion of the density operator a semi-classical relaxation term. They showed that the concept of the geometric phase remains meaningful in the presence of the stochastic process. This approach has, however, an obvious fundamental restriction: it cannot be extended to systems with quantum degeneracies. Furthermore, in non-degenerate quantum systems the line broadening resulting from the stochastic process could mask the geometrical effects. It was concluded, for example, that in an ESR experiment the geometric phase may be observed only in the domain of slow motions [4].

In this Letter we consider the same kind of problem but for systems with degenerate quantum states. The Fano-Zwanzig projection operator method [5] will be used to describe the evolution of the ensemble-averaged propagator $\langle\hat{U}(t)\rangle$. The general expression for the corresponding memory superoperator (or 'cumulant kernel' [6]) in terms of ordered cumulants of a non-Abelian one-form is obtained.

As an application of this general formalism the process of electron spin-rotational (SR) relaxation in linear radicals with $S_{\mathrm{ef}}=1 / 2$ will be briefly consid- 
ered. For this case it will be shown that SR relaxation arises from the stochastic modulation of a WilczekZee non-Abelian gauge potential [7], i.e. it is of 'purely geometric' origin.

\section{General theory}

Consider a cyclic evolution of an $N$-fold degenerate state vector $|\psi\rangle$ under an adiabatic variation of the Hamiltonian, $H[\Omega(t)]$, along the path $\boldsymbol{\Omega}(t)$ in the parameter space. It was shown [7] that if the set of instantaneous eigenstates remains degenerate as the Hamiltonian varies, the evolution of the system is governed by a corresponding non-Abelian one-form $A$. In the Liouville space we may express this as $|\rho(T)\rangle\rangle=\hat{O}(T)|\rho(0)\rangle$, where $|\rho\rangle\rangle=|\psi\rangle \otimes\langle\psi|$ is a density operator, $T$ is the period of a cycle, i.e. the time $T$ when $\Omega(T)=\Omega(0)$ and the propagator $\hat{U}(t)$ satisfies,

$\frac{\mathrm{d}}{\mathrm{d} t} \hat{O}(t)=-\hat{A}(t) \hat{O}(t), \hat{U}(0)=\hat{1}$.

The $(N \times N)^{2}$ anti-Hermitian matrix $\hat{A}$ is defined by

$\hat{A}(t)=|\rho[\Omega(t)]\rangle \otimes\left\langle\left\langle\left(\frac{\mathrm{d}}{\mathrm{d} t} \rho[\Omega(t)]\right)\right|\right.$.

Here, $|\rho[\Omega(t)]\rangle$ is the instantaneous eigenvector of the Liouvillian $H^{\times}[\Omega(t)]$. In the following $\hbar=1$, and the notation $B^{\times} b=[B, b]$ will be used. If the evolution of the quantum system is non-stochastic, Eq. (1) can be immediately integrated to give

$\hat{U}(t)=\exp _{T}\left(-\int_{0}^{t} \hat{A}(\tau) \mathrm{d} \tau\right)$,

where the subscript $T$ specifies time ordering.

For chaotic systems, however, the trajectory in the parameter space has a random character. Suppose that this process is stationary and ergodic, and has the finite correlation time $\tau_{c}$. In this case the usual assumption of the adiabatic approximation $\left\|H^{\times}\right\| \tau_{\mathrm{c}} \gg 1$ is assumed to hold and it is necessary to find the ensemble average of $\hat{U}(t)$. In order to do this we can use the Fano-Zwanzig projection operator method [5] to deduce from Eq. (1) the following kinetic equation for the ensemble averaged, \langle\rangle , propagator: $\frac{\mathrm{d}}{\mathrm{d} t}\langle\hat{U}(t)\rangle=\int_{0}^{t}\left\langle\hat{K}_{G}(t, \tau)\right\rangle\langle\hat{U}(\tau)\rangle \mathrm{d} \tau$,

where the memory superoperator (or 'cumulant kernel' [6]) is

$$
\begin{aligned}
& \left\langle\hat{K}_{G}\left(t, \tau_{1}\right)\right\rangle=\hat{g}_{2}\left(t, \tau_{1}\right) \\
& +\sum_{n=2}^{\infty} \int_{0}^{\tau_{1}} \mathrm{~d} \tau_{2} \ldots \int_{0}^{\tau_{n}-1} \mathrm{~d} \tau_{n} \hat{g}_{n+1}\left(t, \tau_{1}, \ldots, \tau_{n}\right), \\
& \hat{g}_{1}=0, \quad \hat{g}_{2}=\hat{m}_{2}, \quad \hat{g}_{3}=\hat{m}_{3}, \\
& \hat{g}_{4}=\hat{m}_{4}-m_{2}\left(t, \tau_{1}\right) m_{2}\left(\tau_{2}, \tau_{3}\right), \\
& \hat{m}_{n}\left(\tau_{1}, \tau_{2}, \ldots, \tau_{n}\right)=(-1)^{n}\left\langle\hat{A}\left(\tau_{1}\right) \hat{A}\left(\tau_{2}\right) . . \hat{A}\left(\tau_{n}\right)\right\rangle,
\end{aligned}
$$

and it is assumed that $\langle\hat{A}(\tau)\rangle=0$. In the "fast motional' or Redfield limit, i.e. if $\left\|\left\langle\hat{A}^{2}\right\rangle\right\| \tau_{\mathrm{c}}^{2} \ll 1$, to second-order in the cumulant expansion $\left(g_{n}=0, n \geqslant 3\right)$ we get

$$
\langle\hat{U}(t)\rangle=\exp _{T}\left(\int_{0}^{\tau} \mathrm{d} \tau \int_{0}^{\tau} \mathrm{d} \tau_{1}\left\langle\hat{A}(\tau) \hat{A}\left(\tau_{1}\right)\right\rangle\right) .
$$

If the correlation time $\tau_{\mathrm{c}}$ is much shorter than the period of a cycle, $\tau_{\mathrm{c}} \ll T$, the stochastic modulation of the non-Abelian gauge field results in relaxation of the quantum system. Since $\langle\rho(t)\rangle=\langle\theta(t)\rangle \rho(0)$, in the 'fast motional' limit, this process is described by the following kinetic equation:

$\frac{\mathrm{d}}{\mathrm{d} t}\langle\rho(t)\rangle=\int_{0}^{t}\langle\hat{A}(t) \hat{A}(\tau)\rangle\langle\rho(\tau)\rangle \mathrm{d} \tau$.

Non-Abelian gauge potentials can be found in various physical systems [2] where their stochastic modulation will induce different kinds of relaxation processes. The geometrical approach, however, allows one to treat these diverse relaxation phenomena within a single unified framework.

\section{Stochastic modulation of Wilczek-Zee gauge potential}

The remainder of this paper will be devoted to a particular example which is related to observable ef- 
fects of the stochastic modulation of a $U(2)$ Wilczek-Zee gauge potential $A_{\mathrm{wz}}$ [7] on a doubly degenerate quantum system with an axially symmetric Hamiltonian $H$ of the non-rotating system. In the rotating frame of reference (M) attached to the system the Wilczek-Zee one-form is given by [7],

$$
\begin{aligned}
& A(\mathrm{~W})=-\mathrm{i}\left[\dot{\theta}_{\kappa} S_{\mathrm{ef}, y}^{(\mathrm{M})}\right. \\
& \quad+\dot{\phi}\left(-\sin \theta \kappa S_{\mathrm{ef}, x}^{(\mathrm{M})}+\cos \theta S_{\mathrm{ef}, z}^{(\mathrm{M})}\right] .
\end{aligned}
$$

Here, $\kappa$ is a parameter depending on the particular physical situation, defined by $H ;\{\theta, \phi\}=\Omega$ is a set of spherical angles defining the orientation of the $C_{\infty}$ axis $\left(Z_{\mathrm{M}}\right.$ axis of $\left.H\right), S_{\mathrm{ef}, i}=\sigma_{i} / 2$, where $\sigma_{i}$ is the corresponding Pauli matrix. As we have shown elsewhere [8], Eq. (7) can be represented in a more compact and general tensor form:

$A \underset{\mathrm{WZ}}{(\mathrm{M})}=-\mathrm{i} \omega \hat{\gamma} S_{\mathrm{ef}}^{(\mathrm{M})}$,

where $\omega$ denotes the angular velocity describing the rotation of the $\mathrm{M}$ frame. Eq. (7) corresponds to the special case: $\kappa=\hat{\gamma}_{x, x}=\hat{\gamma}_{y, y} \equiv \gamma_{\perp}, \hat{\gamma}_{z, z} \equiv \gamma_{\|}=1$. Note that the origin 'unrotated' Hamiltonian $H$ which comprises the actual physical problem is now hidden in the definition of the $\hat{\gamma}$ 'tensor' (see ref. [8] for details).

Now let us transform $A(\mathrm{MZ}) \mathrm{Eq}$. (8) into the labframe $(L)$,

$A \stackrel{(\mathrm{W})}{\mathrm{W}}=-\mathrm{i} \omega \delta \hat{\gamma} S_{\mathrm{ef}}^{(\mathrm{L})}$,

where $\delta \hat{\gamma}=\hat{\gamma}-\hat{\mathbf{1}}$. This equation shows that in the $L$ frame the response of a doubly degenerate quantum system to an adiabatic variation of $\Omega$ can be described as the interaction of the effective $\operatorname{spin} S_{\text {ef }}=1 /$ 2 with a 'fictitious' magnetic field $f=-\omega \delta \hat{\gamma}$. This coupling is equivalent to the action of an effective spin Hamiltonian $H_{\text {ef, }}$

$H_{\mathrm{ef}}^{(\mathrm{L})}=-\mathrm{i} A{ }_{\mathrm{W} Z}^{(\mathrm{L}}=-\omega \delta \hat{\gamma} S_{\mathrm{ef}}^{(\mathrm{L})}$,

and may be viewed as the origin of $S_{\text {ef }}$ relaxation when $A(\mathrm{WZ}(t)$ has a random character.

It is important to note that the result we arrived at in Eq. (10) is independent of the particular physical problem. Formally, however, it has the same structure as the spin Hamiltonian of spin-rotational interaction for systems with $S_{\mathrm{ef}}=1 / 2[9,10]$, which for a linear rotator can be written as

$H_{\mathrm{sr}}^{(\mathrm{L})}=\omega\left[(2 B)^{-1} \mathrm{C}_{\mathrm{sr}}\right] S_{\mathrm{ef}}^{(\mathrm{L})}$, where $B$ is the rotational constant and $\mathrm{C}_{\mathrm{sr}}$ is the spinrotational tensor. It has been shown in ref. [8] that if, instead of a rather general doubly degenerate quantum system, we consider a linear radical with $S_{\mathrm{ef}}=1 / 2$, then $H_{\mathrm{ef}}^{(\mathrm{L})}$ and $H_{\mathrm{sI}}^{(\mathrm{L})}$ are not only of the same form but become physically equivalent if one defines

$\delta \hat{\gamma} S_{\mathrm{ef}} \equiv P_{\mathrm{KD}} L P_{\mathrm{KD}}+\left(P_{\mathrm{KD}} S P_{\mathrm{KD}}-S_{\mathrm{ef}}\right)$,

where $L$ and $S$ are the operators of electronic orbital momentum and spin, respectively, and $P_{\mathrm{KD}}$ is the projection operator onto the adiabatically isolated, spin-orbit mixed Kramers doublet. The spin-rotational tensor $\mathrm{C}_{\mathrm{sr}}$ and the $\gamma$ tensor defined in Eq. (12) are related by

$\mathrm{C}_{\mathrm{sr}}=-2 B \delta \hat{\gamma}$.

It follows from Eq. (12) that in the absence of spinorbit coupling (SOC) the gauge potential vanishes, and $\mathrm{C}_{\mathrm{sr}}=0$. It was shown [8] that to first-order in SOC $\delta \hat{\gamma}=\delta \hat{g}$, where $\delta \hat{g}=\hat{g}-g_{e} \hat{\mathbf{i}}$, corresponds to the deviation of the components of the $g$ tensor from the value of the free electron. Therefore, in the case of weak SOC, as applies to most organic radicals, Eq. (13) is equivalent to the traditional result [9]

$\hat{\mathrm{C}}_{\mathrm{sr}}=-2 B \delta \hat{g}$.

On the other hand, it must be emphasized that Eq. (13) is exact within the adiabatic limit, whereas Eq. (14) is valid only for $\|\delta \hat{\gamma}\| \ll 1$.

In the case of weak action of the gauge potential $\left(\left\|\hat{A}_{\mathrm{WZ}}^{2}\right\| \tau_{\mathrm{c}}^{2} \ll 1\right)$ it is natural to assume that the relaxation of $\boldsymbol{\Omega}(t)$ proceeds at a much higher rate than the relaxation of $S_{\mathrm{ef}}(t)$. This means that the average state of a quantum system represented by $S_{\mathrm{ef}}(t)$ is independent of the particular position in the parameter space. In this limit the corresponding relaxation time

$T_{s}=\frac{\operatorname{Tr} \int_{0}^{\infty} \mathrm{d} t\left[S_{\mathrm{ef}, Z_{\mathrm{L}}}\langle\rho(t)\rangle\right]}{\operatorname{Tr}\left[S_{\mathrm{ef}, Z_{\mathrm{L}}} \rho(0)\right]}$,

can be easily evaluated from the solution of Eq. (6),

$T_{s}=\frac{\operatorname{Tr}\left[S_{\mathrm{ef}, \mathrm{Z}_{\mathrm{L}}} \hat{R}^{-1} \rho(0)\right]}{\operatorname{Tr}\left[S_{\mathrm{ef}, \mathrm{Z}_{\mathrm{L}}} \rho(0)\right]}$,

where

$\hat{R}=-\int_{0}^{\infty} \mathrm{d} \tau\left\langle\hat{A}_{\mathrm{w} Z}^{(\mathrm{L} Z}(\tau) \hat{A}_{\mathrm{wZ}}^{\mathrm{L} Z}(0)\right\rangle$. 
Thus, in the case of weak action of the Wilczek-Zee gauge potential the study of $S_{\mathrm{er}}(t)$ relaxation is reduced to the traditional calculations of the Redfieldtype relaxation superoperator $\hat{R}$. This conclusion is independent of the particular physical problem and of the particular model of the stochastic process. For dilute liquid solutions of linear radicals the adiabatic condition, $\left\|H^{\times}\right\| \tau_{c} \gg 1$, is fulfilled and the result obtained from Eqs. (16) and (17) is

$$
1 / T_{\mathrm{s}}=\frac{4}{3} \delta \gamma_{\perp}^{2} \tau_{\theta_{1}}^{-1},
$$

where $\tau_{\theta_{1}}$ is the orientational relaxation time of a firstrank tensor (see ref. [10] for details).

We conclude by noting that the formal equivalence of Eq. (1) and the Liouville-von Neumann equation with an effective Liouvillian $H_{\text {ef }}^{\times}=-\mathrm{i} \hat{A}$ allows treatment of the relaxation of a quantum system subject to random modulation of a corresponding gauge potential within the framework of the well-developed stochastic Liouville formalism $[5,6]$. The geometrical approach to the description of the relaxation process is general and the final result can be applied to a variety of physical situations. (It does not depend on a particular physical problem.)

Finally, we wish to point out that within the geometrical concept the quantum state of a system is a unique function of the trajectory in the orientational configuration space. It is independent of the particular values of angular velocity realized on this trajectory. This approach provides a most fruitful basis for a stochastic treatment of systems with non-Abelian gauge field of arbitrary strength [11].

\section{Acknowledgement}

Financial support of this work by the Deutsche Forschungsgemeinschaft and the grant of a Alexander von Humboldt fellowship to YAS is gratefully acknowledged.

\section{References}

[1] M.V. Berry, Proc. Roy. Soc. A 392 (1984) 45.

[2] A. Shapere and F. Wilczek, eds., Geometric phase in physics (Singapore, World Scientific, 1989);

J.W. Zwanziger, M. Koenig and A. Pines, Ann. Rev. Phys. Chem. 41 (1990) 601;

J. Anandan, Nature 360 (1992) 307;

C.A. Mead, Rev. Mod. Phys. 64 (1992) 51.

[3] J.M. Robbins and M.V. Berry, Proc. Roy. Soc. A 436 (1992) 631.

[4] D. Gamliel and J.H. Freed, Phys. Rev. A 39 (1989) 3238.

[5] U. Fano, Phys. Rev. 131 (1963) 259;

R. Zwanzig, Physica 30 (1964) 1109.

[6] R. Kubo, J. Math. Phys. 4 (1963) 174.

[7] F. Wilczek and A. Zee, Phys. Rev. Letters 52 (1984) 2111 ; J. Moody, A. Shapere and F. Wilczek, Phys. Rev. Letters 56 (1986) 893;

A. Zee, Phys. Rev. A 38 (1988) 1.

[8] U.E. Steiner and Yu.A. Serebrennikov, J. Chem. Phys., submitted for publication.

[9] R.F. Curl Jr., J. Chem. Phys. 37 (1962) 779; Mol. Phys. 9 (1965) 585 ;

P.W. Atkins and D. Kivelson, J. Chem. Phys. 44 (1966) 169.

[10] P.S. Hubbard, Phys. Rev. 131 (1963) 1155;

J. McConnell, The theory of nuclear magnetic relaxation in liquids (Cambridge Univ. Press, Cambridge, 1987).

[11] Yu.A. Serebrennikov and U.E. Steiner, J. Chem. Phys., submitted for publication. 